\title{
Narrow Anteroposterior Thorax may be Associated with Brugada Syndrome due to Chronic Mechanical Compression of the Right Ventricular Outflow Tract
}

\author{
Yumi Ishii ${ }^{1}$, Mikiko Nakagawa ${ }^{2}$, Ichitaro Abe ${ }^{3}$, Hidekazu Kondo ${ }^{3}$, Akira Fukui ${ }^{4}$, Hidefumi \\ Akioka $^{1}$, Tetsuji Shinohara ${ }^{5}$, Yasushi Teshima ${ }^{6}$, Kunio Yufu ${ }^{6}$, and Naohiko Takahashi ${ }^{7}$ \\ ${ }^{1}$ Oita university \\ ${ }^{2}$ Oita University, Faculty of Medicine \\ ${ }^{3}$ Oita University \\ ${ }^{4}$ Faculty of Medicine \\ ${ }^{5}$ Indiana University School of Medicine \\ ${ }^{6}$ Oita University, School of Medicine \\ ${ }^{7}$ Faculty of Medicine, Oita University
}

September 10, 2020

\begin{abstract}
Introduction: Recent research has shown that Brugada syndrome $(\mathrm{BrS})$ is associated with interstitial fibrosis in the right ventricular outflow tract (RVOT) epicardium, thus suggesting that BrS does not just involve cardiac channelopathy but also includes cardiomyopathy. On the other hand, Brugada pattern ECGs are often observed in patients with pectus excavatum. The purpose of this study was to investigate whether thoracic deformity, along with mechanical compression of RVOT, were associated with BrS. Methods and Results: We recruited 17 male patients with symptomatic BrS, 32 male patients with asymptomatic $\mathrm{BrS}$, and 30 age-matched male controls. Using computed tomography (CT) scans, we measured the maximal internal transverse diameter of the thorax $(\mathrm{T})$ and the shortest anteroposterior depth from the internal aspect of the sternum to the anterior cortex of the vertebral body (D) at the level of the RVOT. We then evaluated the fragmented QRS (f-QRS) in the right precordial leads. D was significantly shorter, while $\mathrm{T}$ divided by $\mathrm{D}(\mathrm{T} / \mathrm{D})$ was significantly greater, in patients with symptomatic and asymptomatic BrS than in the controls. Six patients with pectus excavatum (T/D[?]3.25) were included in the BrS population. The f-QRSs was more frequently observed in symptomatic and asymptomatic BrS patients than in controls. The positive spikes within the QRS complex were more prevalent in patients with a greater T/D. Conclusion: Our results suggested that a narrow anteroposterior thoracic space could be associated with BrS via mechanical compression of the RVOT.
\end{abstract}

\section{Key words}

Brugada syndrome; fragmented QRS; right ventricular outflow tract; thoracic deformity; pectus excavatum.

\section{Introduction}

Since 1992, Brugada syndrome (BrS) has attracted significant attention as a high risk disease that is associated with sudden cardiac death in young and healthy adults ${ }^{1}$. Typical ST elevations, referred to as the "coved pattern" have been recognized in electrocardiograms from patients in the BrS; the prevalence of this pattern in the healthy population is reported to be $0.1 \%^{2}$. BrS is regarded as an inherited arrhythmia 
syndrome and is thought to involve cardiac channelopathy. However, variants in cardiac ion channel genes such as SCN5A have been identified in only $20-30 \%$ of cases ${ }^{3}$.

On the other hand, in BrS, myocardial fibrosis has been identified as abnormally low voltage or fractionated electrograms that are localized to the right ventricular outflow tract (RVOT) at the epicardium ${ }^{4}$. The ablation of these sites was shown to eliminate the coved ECG pattern and successfully reduce arrhythmic events $^{5}$. Morita et al. further showed that the fragmented QRS (f-QRS) in the right precordial leads represented a depolarization abnormality of the RVOT, which predicted lethal arrhythmic events in patients with $\mathrm{BrS}{ }^{6,7}$.

Brugada ECG patterns are associated with various pathophysiological conditions such as electrolyte abnormalities, myocardial ischemia, pericarditis, myocarditis, pulmonary embolism, and mechanical mediastinal compression. Several case reports have shown that Brugada ECG patterns are observed in patients with pectus excavatum ${ }^{8,9}$. We hypothesized that thoracic deformities such as pectus excavatum may induce a chronic mechanical injury to the RVOT caused by compression of the anterior chest wall and thus play a role in the genesis of Brugada ECG patterns and f-QRS in the right precordial leads. Therefore, in the present study, we investigated whether a narrowing of the thoracic anteroposterior space could be associated with the genesis of BrS.

\section{Methods}

\section{Study population}

Between July 2008 and May 2020, 49 consecutive patients were diagnosed with BrS at Oita University Hospital. BrS was diagnosed according to the criteria put forward by expert consensus statements ${ }^{10}$.

We divided BrS patients into two groups: symptomatic BrS $(\mathrm{n}=17)$ and asymptomatic $\mathrm{BrS}(\mathrm{n}=32)$. All patients with symptomatic BrS experienced syncope or lethal arrhythmic events. Fifteen of the $17 \mathrm{BrS}$ patients received implantable cardioverter defibrillators (ICDs); two patients did not agree to be implanted with an ICD. The patients with asymptomatic BrS did not experience any syncopal events or ventricular arrhythmias.

Thirty age-matched males with normal ECGs were also included as controls. None of the controls had any evidence of organic heart diseases on clinical examination findings, standard 12-lead ECGs, chest X-rays, and echocardiograms.

\section{ECG recording and the definition of f-QRS}

With the subjects resting in a supine position, we recorded standard 12-lead surface digital ECGs using a CardiMax FCP-8800 system (Fukuda Denshi, Tokyo, Japan) with a 150-Hz low-pass filter. The PQ, QRS, and QT intervals were automatically measured using a commercially available program on each ECG. The Fridericia formula was then used to calculate the rate-corrected QT (QTc).

The number of positive spikes within the QRS complex in leads $V_{1}$ to $V_{3}$ were measured manually with amplified waveforms. The f-QRS was defined as the QRS complex with $>2$ positive spikes within the QRS complex in two contiguous leads, in accordance with Morita's new criteria ${ }^{7}$. ECGs recorded prior to the initiation of drug therapy were used for analysis. All ECGs were reviewed by two experienced cardiologists who were blinded to other information.

\section{Evaluation of thorax size by CT scan}

All study subjects had undergone thoracic computed tomography (CT) scans around the same time as the ECG recordings. Thorax size was calculated by the Haller's method ${ }^{11}$. Using the CT scans, two parameters were measured at the level of the RVOT (Figure 1 ). The first parameter was the maximal internal transverse diameter of the thorax $(\mathrm{T})$. The second parameter was the shortest anteroposterior depth (D) measured from the internal aspect of the sternum to the anterior cortex of the vertebral body. The T, D, and $\mathrm{T}$ divided by $\mathrm{D}(\mathrm{T} / \mathrm{D})$, were compared between the three groups (symptomatic BrS, asymptomatic BrS, and 
controls). According to Haller's report ${ }^{11}$, we considered T/D[?]2.5 as normal and $\mathrm{T} / \mathrm{D}[?] 3.25$ to indicate pectus excavatum.

This study protocol was approved by the Ethics Committee of Oita University Hospital (Approval number: 1650) and was conducted in accordance with the guidelines proposed in the Declaration of Helsinki.

\section{Statistics}

Continuous data were evaluated for normality using the Shapiro-Wilk test and expressed as mean $\pm \mathrm{SD}$. The Student's $t$ - test was used for comparisons between groups because all data were normally distributed. Oneway analysis of variance (ANOVA) was used to determine significant differences among the three groups. All statistical tests were performed using JMP version11 (SAS Institute, Cary, NC) using Windows 10 (Microsoft, Redmond, WA). $\mathrm{P}<0.05$ was considered to be statistically significant.

\section{Results}

\section{Patient characteristics}

Clinical characteristics were compared between the three groups (Table 1). Age, body height, body weight, body mass index (BMI), and history of illness, were not significantly different when compared between the three groups. In terms of ECG parameters, heart rate and PR interval were not significantly different between the three groups. However, QRS interval was significantly longer in patients with symptomatic BrS and asymptomatic $\mathrm{BrS}$ than in controls $(\mathrm{P}<0.01$ and $\mathrm{P}<0.05$, respectively). Furthermore, QTc interval was significantly longer in symptomatic patients with $\mathrm{BrS}$ than controls $(\mathrm{P}<0.05)$. There were no significant differences in any echocardiographic findings when compared between the three groups.

\section{Comparison of thoracic size between the three groups}

Thoracic size, as evaluated by CT scans, were compared between the three groups (Figure 2 ). There were no significant differences in the value of $\mathrm{T}$ when compared between symptomatic BrS patients, asymptomatic BrS patients, and controls $(254.4 \pm 10.4,256.1 \pm 14.7$, and $258.0 \pm 11.6 \mathrm{~mm}$, respectively) (Figure 2A ). The value of $\mathrm{D}$ was significantly shorter in patients with symptomatic $\mathrm{BrS}$ and asymptomatic $\mathrm{BrS}$ than in controls (92.9 \pm 17.2 and $98.8 \pm 11.2$ vs $108.7 \pm 14.5 \mathrm{~mm}$, respectively, $\mathrm{P}<0.01)$ (Figure 2B ). The value of $\mathrm{T} / \mathrm{D}$ was significantly greater in patients with symptomatic $\mathrm{BrS}$ and asymptomatic BrS than in controls $(2.85 \pm 0.66$ and $2.62 \pm 0.26$ vs $2.41 \pm 0.31, \mathrm{P}<0.01$ and $\mathrm{P}<0.05$, respectively) (Figure $2 \mathrm{C}$ ). The values of both $\mathrm{D}$ and $\mathrm{T} / \mathrm{D}$ were not significantly different when compared between patients with symptomatic BrS and asymptomatic BrS. Four patients in the symptomatic BrS group and two patients in the asymptomatic BrS group fulfilled the definition of pectus excavatum (T/D[?]3.25).

The representative cases of symptomatic BrS are shown in Figures 3 and $\mathbf{4}$. Case 1 was a 64-year-old male with typical ST-segment elevations on his ECG since he was younger (Figure 3). He suddenly experienced cardiac arrest at home and was transported to the emergency department. His ECG monitor showed several episodes of ventricular fibrillation; these episodes were treated by isoproterenol. He had received subcutaneous ICD implantation after being excluded from organic cardiac diseases by echocardiography, catheter examination, and cardiac magnetic resonance imaging. His ECG showed the coved type pattern in $\mathrm{V}_{1}$ and $\mathrm{V}_{2}$ leads. $\mathrm{T}$ and $\mathrm{D}$, as determined by his $\mathrm{CT}$ scan, were $232.3 \mathrm{~mm}$ and $68.4 \mathrm{~mm}$, respectively. The T/D was 3.40; this satisfied the definition of pectus excavatum. While the depression of his anterior chest wall was not so remarkable, his anteroposterior thoracic space was narrow and the sternum attached to the surface of RVOT.

Case 2 was an 18-year-old high school boy who had a mild pectus excavatum (Figure 4) . He experienced syncope due to ventricular fibrillation at school and was resuscitated with an automated external defibrillator (AED). His coronary angiography showed normal coronary function and an ergonovine provocation test was negative. A subcutaneous ICD was implanted for secondary prevention of sudden cardiac death. He had the coved type pattern in $V_{1}$ and a superior intercostal space of $V_{1}-V_{2}$ leads. The f-QRS was observed in 
$\mathrm{V}_{1}$ and $\mathrm{V}_{2}$ leads. $\mathrm{T}, \mathrm{D}$, and $\mathrm{T} / \mathrm{D}$, were $262.0 \mathrm{~mm}, 54.7 \mathrm{~mm}$, and 4.79 , as determined by $\mathrm{CT}$ scan, and his RVOT was mildly compressed by the deformed anterior chest wall.

\section{Prevalence of f-QRS}

Next, we evaluated the prevalence of f-QRS in the right precordial leads in each group. A representative example of f-QRS is shown inFigure 5A . The prevalence of $\mathrm{f}-\mathrm{QRS}$ was significantly higher in patients with symptomatic $\mathrm{BrS}$ and asymptomatic $\mathrm{BrS}$ than in controls $(41 \%, 34 \%$ and $3 \%$, respectively, $\mathrm{P}<0.01$ ) (Figure 5B ). In addition, the total number of positive spikes within the QRS complex in the right precordial leads were evaluated with regards to T/D value: [?]2.5 $(\mathrm{n}=16), 2.5$ to $3.25(\mathrm{n}=27)$, and [?] $3.25(\mathrm{n}=6)$ (Figure 5C ). The total number of positive spikes within the QRS complex was significant greater in patients with $\mathrm{T} / \mathrm{D}[?] 3.25$ and 2.5 to 3.25 than in patients with a normal T/D ([?]2.5) (7.2+-2.1 and $6.4+-1.7$ vs 5.3+-1.9, respectively, $\mathrm{P}<0.05)$.

\section{Discussion}

To the best of our knowledge, this is the first study to address a potential association between thoracic deformity, a narrow anteroposterior thoracic space, and BrS. The main findings of this study were as follows: (1) the value of $\mathrm{D}$ was significantly shorter, and $\mathrm{T} / \mathrm{D}$ was significantly greater, in patients with symptomatic and asymptomatic BrS than in the controls; and (2) the f-QRS in the right precordial leads was observed more frequently in patients with BrS than in controls. The positive spikes within the QRS complex were more prevalent in patients with the greater $\mathrm{T} / \mathrm{D}$.

\section{Pathophysiology of $\mathrm{BrS}$}

BrS has been regarded as an inherited life-threatening disease. However, genetic mutations are only detected in around $20-30 \%$ of patients ${ }^{3}$. Recent findings suggested that $\mathrm{BrS}$ included not only genetic cardiac channelopathy, but also secondary cardiomyopathy. Nademanee et al. and Pappone et al. showed that the arrhythmogenic electrophysiological substrate was characterized by low voltage and prolonged fractionated late potentials clustering in the anterior aspect of the RVOT epicardium in patients with BrS. Their elimination by radiofrequency ablation resulted in normalization of the Brugada ECG pattern and prevention of ventricular fibrillation recurrence in $\mathrm{BrS}^{4,5,12}$.

The existence of f-QRS could help us to explain the pathophysiological mechanisms underlying BrS, with regards to repolarization abnormalityvs depolarization abnormality. The f-QRS in the right precordial leads suggested depolarization abnormalities at the RVOT. Morita et al. reported that f-QRS was observed in $43 \%$ of patients with symptomatic $\mathrm{BrS}^{6}$; this was consistent with our current data (41\%). In this study, QRS and QTc intervals were significantly longer in patients with symptomatic BrS and asymptomatic BrS than in controls.

\section{Relationship between thoracic size and $\mathrm{BrS}$}

The value of $\mathrm{D}$ was significantly shorter, and $\mathrm{T} / \mathrm{D}$ was significantly greater, in the patients with symptomatic and asymptomatic BrS than in controls. In Haller's report ${ }^{11}$, normal controls had a $\mathrm{T} / \mathrm{D}<2.56$; this was consistent with the mean value of $\mathrm{T} / \mathrm{D}$ in our current controls $(2.41+-0.31)$. In total, six patients with pectus excavatum (T/D[?]3.25) had BrS; four had symptomatic BrS and two had asymptomatic BrS. Pectus excavatum is a common congenital chest wall deformity in which the sternum is depressed posteriorly; this can cause severe cardiac compression and displacement. However, patients with a flat anterior chest wall, or mildly inward deformation of the sternum, such as case 1, should also be classified as having pectus excavatum according to Haller's criteria (T/D[?]3.25). Therefore, we treated patients with not only typical pectus excavatum but also a short anteroposterior depth as patients with a narrow thorax in this study.

A narrow thorax may directly affect the surface of the RVOT; this is because the RVOT commonly exists just behind the sternum. Chronic mechanical compression may lead to fibrosis and abnormal conduction in the epicardial side at the RVOT. The electrical gradient between the epicardium and the endocardium in the RVOT may result in ST elevation and conduction disturbance may appear as f-QRS in the right precordial 
leads; these are identical with the typical ECGs in $\mathrm{BrS}^{13}$. Interestingly, the number of spikes within the QRS complex in the right precordial leads was greater in patients with a greater T/D (Figure 5C ), thus supporting our hypothesis.

A narrow thorax may explain several cardiac issues. Several case reports have reported the existence of Brugada ECG patterns in patients with a narrow thoracic deformity, including pectus excavatum ${ }^{9,14,15}$, anterior mediastinal masses ${ }^{16}$, and the surgical treatment of esophageal cancer followed by retro-sternum placement of the stomach ${ }^{17}$.

Ahn et al. reported a young male with pectus excavatum who presented a coved pattern in the $V_{2}$ to $V_{3}$ leads ${ }^{14}$. His ST-segment morphology was normalized following surgery to fix the thoracic deformity. Sudden cardiac arrests have also been reported in young males with pectus excavatum ${ }^{18,19}$.

On the other hand, Tran et al. reported that the mean T/D was significantly higher in patients with lone atrial fibrillation (AF) when compared to patients with non-lone $\mathrm{AF}$ and controls ${ }^{20}$. Pectus excavatum can cause right atrial and RVOT compression against the sternum and left atrial compression against the spinal column. Mechanical compression may lead to fibrosis in both atria resulting in a substrate for AF. Interestingly, patients with $\mathrm{BrS}$ are frequently complicated by $\mathrm{AF}^{21}$.

Furthermore, the incidence of pectus excavatum is 4 -fold higher in males than females ${ }^{22}$; this is consistent with the male dominance of BrS. In addition, BrS is strongly associated with a family history; furthermore, $43 \%$ of patients with pectus excavatum also have a family history ${ }^{23}$. Together with these findings, there were various commonalities between $\mathrm{BrS}$ and narrow thoracic deformities. BrS is known to be a heterogeneous disease. The association between thoracic deformity and $\mathrm{BrS}$ may partially explain the mechanisms that underlie the genesis of BrS. However, we did not find any significant differences in thoracic size when compared between symptomatic BrS and asymptomatic BrS. Further depolarization and/or repolarization abnormalities in RVOT may be closely associated with arrhythmogenesis in symptomatic BrS ${ }^{24}$.

Further studies are now needed to corroborate this association and elucidate the potential mechanisms underlying the pathogenesis of BrS.

\section{Limitations}

Some limitations existed in this study that should be considered. First, because this study was performed retrospectively in a single center, the number of patients was limited. Second, we did not analyze other markers associated with the risk of sudden cardiac death or fatal arrhythmias, such as late potential, early repolarization pattern, T-wave alternance, or gene mutation. This was because we focused on the relationship between BrS and a narrow thorax in this study. Finally, we were unable to provide supporting evidence from imaging modalities, such as cardiac magnetic resonance imaging and electrical three-dimensional mapping.

\section{Conclusion}

Our results demonstrated that a narrow anteroposterior thoracic space was associated with BrS. Therefore, some aspects of BrS could be acquired by the mechanical compression of the RVOT. A prospective and multicenter cohort study is now required to confirm these findings in the future.

\section{References}

1. Brugada P, Brugada J. Right bundle branch block, persistent ST segment elevation and sudden cardiac death: a distinct clinical and electrocardiographic syndrome. A multicenter report. J Am Coll Cardiol. 1992;20(6):1391-1396.

2. Hermida JS, Lemoine JL, Aoun FB, Jarry G, Rey JL, Quiret JC. Prevalence of the brugada syndrome in an apparently healthy population. Am J Cardiol. 2000;86(1):91-94.

3. Nademanee K, Raju H, de Noronha SV, et al. Fibrosis, Connexin-43, and Conduction Abnormalities in the Brugada Syndrome. J Am Coll Cardiol. 2015;66(18):1976-1986. 
4. Nademanee K, Veerakul G, Chandanamattha P, et al. Prevention of ventricular fibrillation episodes in Brugada syndrome by catheter ablation over the anterior right ventricular outflow tract epicardium. Circulation. 2011;123(12):1270-1279.

5. Pappone C, Brugada J, Vicedomini G, et al. Electrical Substrate Elimination in 135 Consecutive Patients With Brugada Syndrome. Circ Arrhythm Electrophysiol. 2017;10(5):e005053.

6. Morita H, Kusano KF, Miura D, et al. Fragmented QRS as a marker of conduction abnormality and a predictor of prognosis of Brugada syndrome.Circulation. 2008;118(17):1697-1704.

7. Morita H, Watanabe A, Morimoto Y, et al. Distribution and Prognostic Significance of Fragmented QRS in Patients With Brugada Syndrome.Circ Arrhythm Electrophysiol. 2017;10(3).

8. Awad SF, Barbosa-Barros R, Belem Lde S, et al. Brugada phenocopy in a patient with pectus excavatum: systematic review of the ECG manifestations associated with pectus excavatum. Ann Noninvasive Electrocardiol. 2013;18(5):415-420.

9. Kataoka H. Electrocardiographic patterns of the Brugada syndrome in 2 young patients with pectus excavatum. J Electrocardiol.2002;35(2):169-171.

10. Sieira J, Brugada P. The definition of the Brugada syndrome.Eur Heart J. 2017;38(40):3029-3034.

11. Haller JA, Jr., Kramer SS, Lietman SA. Use of CT scans in selection of patients for pectus excavatum surgery: a preliminary report. J Pediatr Surg. 1987;22(10):904-906.

12. Nademanee K, Hocini M, Haissaguerre M. Epicardial substrate ablation for Brugada syndrome. Heart Rhythm. 2017;14(3):457-461.

13. Antzelevitch C, Yan GX. J wave syndromes. Heart Rhythm.2010;7(4):549-558.

14. Ahn J, Choi JI, Shim J, Lee SH, Kim YH. Right Ventricular Compression Mimicking Brugada-Like Electrocardiogram in a Patient with Recurrent Pectus Excavatum. Case Rep Cardiol. 2017;2017:3047937.

15. Marchetti M, Sierecki M, Oriot D, Ghazali A. Brugada-type ECG associated with pectus excavatum. Images Paediatr Cardiol.2015;17(3):1-2.

16. Nakazato Y, Ohmura T, Shimada I, Daida H. Brugada-like precordial ST elevation on ECG by anterior mediastinal infective mass lesion.Indian Pacing Electrophysiol J. 2003;3(3):184.

17. Sasaki A, Nakazato Y. Brugada-like electrocardiogram detected after reconstructive operation for oesophageal cancer. Europace.2010;12(11):1542.

18. Hamoud A, Barry J. Subcutaneous implantable cardioverter defibrillator in a patient with pectus excavatum. Pacing Clin Electrophysiol. 2013;36(5):e138-139.

19. Rachwan RJ, Purpura AK, Kahwash BM. Sudden Cardiac Arrest in a Young Patient With Severe Pectus Excavatum. Am J Med Sci.2018;356(6):570-573.

20. Tran NT, Larry Klein J, Paul Mounsey J, et al. Lone atrial fibrillation is associated with pectus excavatum. Heart Rhythm.2013;10(9):1263-1269.

21. Francis J, Antzelevitch C. Atrial fibrillation and Brugada syndrome.J Am Coll Cardiol. 2008;51(12):11491153.

22. Cartoski MJ, Nuss D, Goretsky MJ, et al. Classification of the dysmorphology of pectus excavatum. $J$ Pediatr Surg.2006;41(9):1573-1581.

23. Abid I, Ewais MM, Marranca J, Jaroszewski DE. Pectus Excavatum: A Review of Diagnosis and Current Treatment Options. J Am Osteopath Assoc. 2017;117(2):106-113. 
24. Pieroni M, Notarstefano P, Oliva A, et al. Electroanatomic and Pathologic Right Ventricular Outflow Tract Abnormalities in Patients With Brugada Syndrome. J Am Coll Cardiol. 2018;72(22):2747-2757.

\section{Figure legends}

Figure 1. Measurements of thoracic size. Two distances at the level of the right ventricular outflow tract (RVOT) were measured from computed tomography (CT) scan; (T) maximal internal transverse diameter of the thorax, (D) shortest anteroposterior depth from the internal aspect of the sternum to the anterior cortex of the vertebral body. In this scan, recorded from a control subject, there was a small space between the sternum and the surface of the RVOT.

Figure 2. Comparison of thoracic size between the three groups. A. Maximal internal transverse diameter of the thorax $(\mathrm{T})$ was not significantly different when compared between the three groups.B. The shortest anteroposterior depth from the internal aspect of the sternum to the anterior cortex of the vertebral body (D) was significantly shorter in patients with symptomatic BrS and asymptomatic BrS than in controls. C. T divided by $\mathrm{D}(\mathrm{T} / \mathrm{D})$ was significantly greater in patients with symptomatic BrS and asymptomatic BrS than in controls. Six patients with BrS fulfilled the definition of pectus excavatum (T/D[?]3.25) (red symbols). The mean values are shown as blue lines.

Figure 3. Representative case of symptomatic Brugada syndrome (case 1). A . ECG showed the coved type pattern in $\mathrm{V}_{1}$ and $\mathrm{V}_{2}$ leads. $\mathrm{B}$. T, D, and T/D, as determined by CT scans, were $232.3 \mathrm{~mm}$, $68.4 \mathrm{~mm}$ and 3.40, respectively. C . The chest x-ray (lateral R-L view) showed a flat anterior chest wall. T; Maximal internal transverse diameter of the thorax, D; Shortest anteroposterior depth from the internal aspect of the sternum to the anterior cortex of the vertebral body.

Figure 4. Representative case of symptomatic Brugada syndrome (case 2). A . ECG showed the coved type pattern in $V_{1}$ and a superior intercostal space of $V_{1}-V_{2}$ leads $\left(^{*}\right)$. The fragmented QRS was observed in $\mathrm{V}_{1}$ and $\mathrm{V}_{2}$ leads (red allows). B . T, D, and T/D, as determined by CT scans, were $262.0 \mathrm{~mm}$, $54.7 \mathrm{~mm}$ and 4.79 , respectively. $\mathbf{C}$. The chest x-ray (lateral R-L view) showed a mild pectus excavatum. T; Maximal internal transverse diameter of the thorax, D; Shortest anteroposterior depth from the internal aspect of the sternum to the anterior cortex of the vertebral body.

Figure 5. Fragmented QRS (f-QRS) on ECGs. A. Representative f-QRS negative and positive ECGs. Red arrows indicate positive spikes within the QRS complex. B. The prevalence of f-QRS was significantly higher in patients with symptomatic BrS and asymptomatic BrS than in controls. C. Box-plot diagram showing the total number of positive spikes within the QRS complex. Minimum and maximum numbers of spikes are depicted as lower and upper bar. The box signifies the upper and lower quartiles, and the median and mean are represented by inside black line and cross symbol, respectively. The total number of positive spikes within the QRS complex was significantly greater in patients with $\mathrm{T} / \mathrm{D}>2.5$ than patients with normal T/D ([?]2.5).

Table1

\begin{tabular}{lllll}
\hline Patient Characteristics & Patient Characteristics & Patient Characteristics & Patient Characteristics \\
\hline & $\begin{array}{l}\text { Symptomatic BrS } \\
(\mathrm{n}=17)\end{array}$ & $\begin{array}{l}\text { Asymptomatic BrS } \\
(\mathrm{n}=32)\end{array}$ & $\begin{array}{l}\text { Controls } \\
(\mathrm{n}=30)\end{array}$ & $P$ \\
Age (years) & $49.3 \pm 17.1$ & $55.1 \pm 14.7$ & $54.1 \pm 16.7$ & 0.47 \\
Body height $(\mathrm{cm})$ & $166.6 \pm 6.4$ & $169.1 \pm 4.9$ & $168.9 \pm 6.4$ & 0.35 \\
Body weight (kg) & $60.4 \pm 12.9$ & $64.2 \pm 12.4$ & $66.9 \pm 13.6$ & 0.26 \\
BMI $\left(\mathrm{kg} / \mathrm{m}^{2}\right)$ & $21.7 \pm 3.8$ & $22.5 \pm 3.9$ & $23.4 \pm 3.9$ & 0.35 \\
History of illness & & $8(26)$ & $8(27)$ & 0.76 \\
Hypertension (\%) & $3(18)$ & $5(16)$ & $5(17)$ & 0.20 \\
$\begin{array}{l}\text { Diabetes mellitus (\%) } \\
\text { Dyslipidemia (\%) }\end{array}$ & 0 & $6(19)$ & $4(13)$ & 0.81
\end{tabular}




\begin{tabular}{lllll}
\hline Patient Characteristics & Patient Characteristics & Patient Characteristics & Patient Characteristics \\
\hline Heart rate (bpm) & $66.6 \pm 6.8$ & $65.7 \pm 10.6$ & $69.6 \pm 9.0$ & 0.24 \\
PR (ms) & $166.1 \pm 27.4$ & $172.2 \pm 26.5$ & $163.1 \pm 23.2$ & 0.37 \\
QRS (ms) & $112.9 \pm 23.8^{* *}$ & $103.7 \pm 11.8^{*}$ & $98.2 \pm 8.4$ & $<0.01$ \\
QTc (ms) & $423.6 \pm 50.2^{*}$ & $402.4 \pm 27.4$ & $403.1 \pm 15.9$ & $<0.05$ \\
Echocardiography & & & & \\
LAD (mm) & $31.0 \pm 8.9$ & $33.8 \pm 5.5$ & $33.0 \pm 6.0$ & 0.44 \\
LVDd (mm) & $47.8 \pm 6.2$ & $47.0 \pm 5.4$ & $46.0 \pm 3.5$ & 0.52 \\
EF (\%) & $64.8 \pm 8.2$ & $64.7 \pm 5.2$ & $66.9 \pm 6.0$ & 0.45 \\
E/e' & $9.0 \pm 6.2$ & $8.7 \pm 2.7$ & $9.4 \pm 3.3$ & 0.84 \\
\hline
\end{tabular}

Data are expressed as mean $\pm \mathrm{SD}$ or $\mathrm{n}(\%)$.

$\mathrm{BrS}=$ Brugada syndrome; $\mathrm{BMI}=$ body mass index; $\mathrm{ECG}=$ electrocardiogram; LAD = left atrial diameter; $\mathrm{LVDd}=$ left ventricular end-diastolic diameter; $\mathrm{EF}=$ ejection fraction; $\mathrm{E} / \mathrm{e}=$ the ratio of $\mathrm{E}$-wave of mitral valves to e-wave at the level of the interventricular septum.

${ }^{*} \mathrm{p}<0.05$ and ${ }^{*} \mathrm{p}<0.01$ vs. controls.

\section{Hosted file}

Figures0901.pptx available at https://authorea.com/users/355461/articles/478681-narrowanteroposterior-thorax-may-be-associated-with-brugada-syndrome-due-to-chronicmechanical-compression-of-the-right-ventricular-outflow-tract 